\title{
A Generalized Oblique Projection Filter with Flexible Parameter for Interference Suppression
}

\author{
Yi-ming Wang, ${ }^{1,2}$ Xing-peng Mao, ${ }^{1}$ Hong Hong, ${ }^{1}$ Jie Zhang, ${ }^{2}$ and Yu-mei Cui ${ }^{1}$ \\ ${ }^{1}$ School of Electronics and Information Engineering, Harbin Institute of Technology, Harbin, Heilongjiang 150001, China \\ ${ }^{2}$ The First Institute of Oceanography, SOA, Qing Dao, Shandong 266061, China \\ Correspondence should be addressed to Xing-peng Mao; mxp@hit.edu.cn
}

Received 25 April 2014; Accepted 20 October 2014

Academic Editor: Michelangelo Villano

Copyright (c) 2015 Yi-ming Wang et al. This is an open access article distributed under the Creative Commons Attribution License, which permits unrestricted use, distribution, and reproduction in any medium, provided the original work is properly cited.

\begin{abstract}
A generalized oblique projection (GOP) with an adjustable parameter defined as interference suppression cost (ISC) is proposed. Therefore, an optional optimized signal to interference-plus-noise ratio (SINR) and user controlled actions on the interference filtering are presented in this GOP framework. Theoretical analysis and numerical simulation demonstrate that when the ISC is derived from minimum variance distortionless response (MVDR) algorithm, the SINR performance of GOP filter is better than both MVDR and oblique projection (OP) filters. Further, an application of GOP filter in ionospheric clutter cancellation in a high frequency surface wave radar (HFSWR) system is given. The ISC is designed specifically to introduce an extra coherent loss to the clutters and a satisfying clutter suppression result is achieved. Besides the examples given, more designs of GOP filter can be inspired by the flexibility of ISC. As a generalized form of OP filter, GOP filter expands the connotation of oblique projection based technique and could be used in spatial filtering, polarization filtering, and other array signal processing applications.
\end{abstract}

\section{Introduction}

As an effective subspace based method, oblique projection [13 ] is used to project measurements into a low-rank subspace along a direction that is oblique to the subspace. In fact, this kind of method is an extension of the widely used orthogonal projection and has been applied in radars [46], communications [7,8], navigations [9], image processing $[10]$, and many other areas $[11,12]$. The subspaces of the oblique projection processed can be either orthogonal or nonorthogonal, which relaxed the orthogonal requirement between subspaces.

The advantage of the oblique projection in retaining the target signals and mitigating the interference has been a promising technique and greatly extended its applications in array signals processing. The oblique projection utilizes the difference between the desired and unwanted signals to extract the target while suppressing interference. As the array, which is formed by a group of sensors sited in a predetermined pattern, generates a featured directional steering vector, spatial information has been used in constructing an oblique projection operator [13-16]. Except for the spatial information, polarization information is also explored in oblique projection to distinguish the target and interference $[4,17]$. Conventional oblique projection based filtering techniques focus on eliminating the interference completely, which may imply a maximized signal to interference ratio (SIR).

One of the problems with the emphasis of maximizing SIR output is that the target signal may be distorted [18]. The oblique projection can resolve it well, whereas the other problem is that when the angle separation between subspaces is small, the noise will be amplified, which leads to a loss in signal to interference-plus-noise ratio (SINR). One way to tackle this problem is to expand single processing domain to multiple domains. When more domains are cooperated, the angle separation can be improved. A collaborative rangeangle-polarization filter [19], which takes advantage of the differences between the signal and interferences in the joint domains, is constructed based on the vector sensitive array.

However, when the target and interference are quite close in multidomain, the angle separation of subspaces is still 
small. To fundamentally solve the problem, a generalized oblique projection (GOP) filter is proposed based on the further research of the traditional oblique projection (OP) operator. Theoretical analysis and simulation results demonstrate that the GOP filter has an improved SINR output capability. Some of the unique properties of the GOP and the flexibilities of interference suppression cost (ISC) by using conventional adaptive signal processing technologies [20,21] or being designed according to a specific high frequency radar application are also explored.

The remaining sections of this paper are organized as follows. In Section 2, the linear model, especially its explicit form in two scenarios of array spatial and polarization filtering, is presented. It is followed by a brief introduction of the OP filter and short analysis of its SINR performance. In Section 3, the generalized form of GOP operator is derived. Four fundamental properties with proofs are given. In Section 4, the spatial GOP is derived and the SINR performance of GOP, OP, and MVDR is evaluated followed by numerical simulations. In Section 5, the GOP filter in polarization domain is discussed and practical application in the ionospheric clutter cancellation problem of a high frequency radar system is demonstrated. Finally, the last section concludes the present work in this paper.

\section{Array Received Signal Model and Oblique Projection Filter}

2.1. The Linear Model. As the signals are characterized as a weighted sum of modes, a linear algebraic framework is applied to vector-valued signals such as those obtained from a sensor array [1]. Assume there are full rank matrices $\mathbf{A} \in \mathbf{S}^{k \times m}$ and $\mathbf{B} \in \mathbf{H}^{k \times t}$ with their columns being linearly independent. The subspaces spanned by them are hence disjoint (unnecessarily orthogonal), which are composed of $k$ observations and $m$ or $t$ parameters. Also, when the additive Gaussian white noise $\mathbf{n}$ is considered, the received linear signal model can be described as

$$
\mathbf{x}=\mathbf{A} \omega+\mathbf{B} \vartheta+\mathbf{n}
$$

where $\omega, \vartheta$ represent mode weights for vectors $\mathbf{A}$ and $\mathbf{B}$, respectively.

The linear model presented here is based on the concept of subspaces, which are not required to be orthogonal. In practical scenarios, spatial subspaces and polarization subspaces can be constructed.

Scenario 1: Array Signal Model in Spatial Domain. The signals received by a uniform linear array (ULA) are a mixture of desired target echoes and interferences from various spatial directions. Under far field assumption, the received signal of the $N$ element array can be described as

$$
\mathbf{x}(t)=\mathbf{a}_{\theta_{1}, \varphi_{1}} \cdot s(t)+\sum_{m=2}^{M-1} \mathbf{a}_{\theta_{m}, \varphi_{m}} \cdot i(t)+\mathbf{n}(t),
$$

where the spatial steering vector of $m$ th signal is given by

$$
\begin{aligned}
& \mathbf{a}_{\theta_{m}, \varphi_{m}} \\
& \quad=\left[\begin{array}{llll}
1 & e^{-j 2 \pi d \sin \theta_{m} \sin \varphi_{m} f / c} & \cdots & e^{-j 2 \pi(N-1) d \sin \theta_{m} \sin \varphi_{m} f / c}
\end{array}\right]^{T}
\end{aligned}
$$

with $\theta, \varphi$ denoting respective azimuth and pitch angles and $d$ being the distance between adjacent sensor elements.

The subspaces spanned by column vector $\mathbf{a}_{\theta_{1}, \varphi_{1}}$ are defined as the target subspace with the description of $\left\langle\mathbf{a}_{\theta_{1}, \varphi_{1}}\right\rangle$ and $\left\langle\mathbf{a}_{\theta_{2}, \varphi_{2}} \cdots \mathbf{a}_{\theta_{m}, \varphi_{m}}\right\rangle$ is defined as interference subspace, correspondingly.

Scenario 2: Array Signal Model in Polarization Domain. In a polarization sensor with biorthogonal polarized antennas, the target subspace and the interference subspace are spanned by their polarization vectors marked by $\mathbf{P}_{\mathbf{s}}$ and $\mathbf{P}_{\mathbf{i}}$, respectively. The signals received can therefore be described as

$$
\mathbf{x}(t)=\mathbf{P}_{\mathbf{s}} s(t)+\mathbf{P}_{\mathbf{i}} i(t)+\mathbf{n}(t),
$$

where $\mathbf{P}_{\mathbf{s}}=\left[\begin{array}{lll}\cos \gamma_{s} & \sin \gamma_{s} e^{j \eta_{s}}\end{array}\right]^{T}, \mathbf{P}_{\mathbf{i}}=\left[\begin{array}{lll}\cos \gamma_{i} & \sin \gamma_{i} e^{j \eta_{i}}\end{array}\right]^{T}$, and $\gamma_{s}, \gamma_{i}$ and $\eta_{s}, \eta_{i}$ depict the respective amplitude relationship and the phase difference between orthogonal electric fields of the target signal and interference.

2.2. Oblique Projection Filter. Consider two full-columnrank complex matrices $\mathbf{A} \in \mathbf{S}^{n \times m}$ and $\mathbf{B} \in \mathbf{H}^{n \times k}$ with $m+k \leq$ $n$; then the columns of $\mathbf{A}$ and $\mathbf{B}$ are both linearly independent and disjoint. Let $\langle\mathbf{A}\rangle$ and $\langle\mathbf{B}\rangle$ denote the subspaces spanned by the columns of $\mathbf{A}$ and $\mathbf{B}$, respectively; then the oblique projection operator $\mathbf{E}_{\mathbf{A B}}$ onto $\mathbf{A}$ along $\mathbf{B}$ is defined as

$$
\mathbf{E}_{\mathbf{A B}}=\mathbf{A}\left(\mathbf{A}^{H} \mathbf{P}_{\mathbf{B}}^{\perp} \mathbf{A}\right)^{-1} \mathbf{A}^{H} \mathbf{P}_{\mathbf{B}}^{\perp}
$$

where $\mathbf{P}_{\mathbf{B}}^{\perp}$ is

$$
\mathbf{P}_{\mathbf{B}}^{\perp}=\mathbf{I}-\mathbf{B}\left(\mathbf{B}^{H} \mathbf{B}\right)^{-1} \mathbf{B}^{H}
$$

Then $\mathbf{E}_{\mathbf{A B}}$ has the following properties:

$$
\begin{aligned}
& \mathbf{E}_{\mathrm{AB}}^{2}=\mathrm{E}_{\mathrm{AB}}, \\
& \mathrm{E}_{\mathrm{AB}} \mathrm{A}=\mathbf{A}, \\
& \mathrm{E}_{\mathrm{AB}} \mathrm{B}=0 .
\end{aligned}
$$

Therefore, we have the output of OP filter as

$$
\begin{aligned}
\widehat{\mathbf{x}}_{\mathrm{OP}}(t) & =\mathbf{E}_{\mathbf{A B}} \mathbf{x}(t)=\mathbf{E}_{\mathbf{A B}}(\mathbf{A} s(t)+\mathbf{B} i(t)+\mathbf{n}(t)) \\
& =\mathbf{A} s(t)+\mathbf{E}_{\mathbf{A B}} \mathbf{n}(t)
\end{aligned}
$$

The scalar form of the OP filter output is

$$
\widehat{s}_{\mathrm{OP}}(t)=\frac{\mathbf{A}^{H}}{\|\mathbf{A}\|^{2}} \mathbf{E}_{\mathbf{A}, \mathbf{B}} \mathbf{x}(t)=s(t)+\frac{\mathbf{A}^{H}}{\|\mathbf{A}\|^{2}} \mathbf{E}_{\mathbf{A}, \mathbf{B}} \mathbf{n}(t) .
$$


The OP operator recovers the desired signal and eliminates interference completely. Therefore, the SINR of the output can be expressed as

$$
\begin{aligned}
\operatorname{SINR} & =\frac{P_{s}}{P_{i}+P_{n}}=\frac{E\left\{\left|\mathbf{A}^{H} \mathbf{E}_{\mathbf{A B}} \mathbf{A} s(t) /\|\mathbf{A}\|\right|^{2}\right\}}{E\left\{\left|\mathbf{A}^{H} \mathbf{E}_{\mathbf{A B}} \mathbf{n}(t) /\|\mathbf{A}\|\right|^{2}\right\}} \\
& =\frac{\sigma_{s}^{2}\|\mathbf{A}\|^{2}}{\sigma_{n}^{2} \operatorname{trace}\left\{\mathbf{E}_{\mathbf{A B}} \mathbf{E}_{\mathbf{A B}}^{H}\right\}}=\frac{\sigma_{s}^{2}}{\sigma_{n}^{2}}\|\mathbf{A}\|^{2} \sin ^{2} \psi,
\end{aligned}
$$

where $\sigma_{s}^{2}, \sigma_{n}^{2}$ stand for the power of target and additive noise, respectively, and the principle angle between $\mathbf{A}$ and $\mathbf{B}$ is defined as

$$
\psi=\arccos \left(\frac{\left\|\mathbf{A}^{H} \mathbf{B}\right\|}{\|\mathbf{A}\|\|\mathbf{B}\|}\right) .
$$

As observed from (8), a portion of noise is retained, mixed with the target signal. The noise power after filtering is given by

$$
P_{n}=E\left\{\left\|\mathbf{E}_{\mathrm{AB}} \mathbf{n}(t)\right\|^{2}\right\}=\frac{1}{\sin ^{2}(\psi)} \sigma_{n}^{2} .
$$

From (9) and (12), we can observe that the noise power after filtering is closely related to the principle angle. When subspaces $\langle\mathbf{A}\rangle$ and $\langle\mathbf{B}\rangle$ get close, which implies a small principle angle, the noise power will be amplified. In this situation, although the interference is totally eliminated, the enhanced noise power may result in a significant SINR loss. This is an inevitable problem of the OP filter.

\section{Generalized Oblique Projection Filter}

The OP filter focuses on the suppression of interference and lacks of the control of the noise power. To obtain a flexible control of the power of the noise and interference simultaneously, a novel oblique projection is proposed, which is named GOP.

3.1. Generalized Oblique Projection Operator. Taking the noise into consideration and introducing an ISC of $\zeta$, the $\mathrm{GOP}$ operator can be derived by solving the following linear constrained optimum problem as

$$
\begin{array}{ll}
\min & \mathbf{w}^{H} \mathbf{w} \\
\text { s.t. } & \mathbf{w}^{H} \mathbf{A}=1 \\
& \mathbf{w}^{H} \mathbf{B}=\zeta,
\end{array}
$$

where $\mathbf{A}$ and $\mathbf{B}$ are the vectors that span target subspace and interference subspace.

From the constraint equation in (13), we have

$$
\mathbf{w}^{H} \mathbf{B}-\zeta \mathbf{w}^{H} \mathbf{A}=\mathbf{w}^{H}(\mathbf{B}-\zeta \mathbf{A})=0
$$

which implicates $\mathbf{B}-\zeta \mathbf{A} \in\langle\mathbf{w}\rangle^{\perp}$.
Also, we have

$$
\begin{aligned}
\mathbf{w}^{H} \mathbf{A} & =\mathbf{w}^{H}\left(\mathbf{P}_{(\mathbf{B}-\zeta \mathbf{A})} \mathbf{A}+\mathbf{P}_{(\mathbf{B}-\zeta \mathbf{A})}^{\perp} \mathbf{A}\right) \\
& =\mathbf{w}^{H} \mathbf{P}_{(\mathbf{B}-\zeta \mathbf{A})}^{\perp} \mathbf{A}=1,
\end{aligned}
$$

where the orthogonal projection operator of $\mathbf{B}-\zeta \mathbf{A}$ is defined as

$$
\begin{aligned}
\mathbf{P}_{(\mathbf{B}-\zeta \mathbf{A})}^{\perp}= & \mathbf{I}-(\mathbf{B}-\zeta \mathbf{A}) \\
& \cdot\left[(\mathbf{B}-\zeta \mathbf{A})^{H} \cdot(\mathbf{B}-\zeta \mathbf{A})\right]^{-1}(\mathbf{B}-\zeta \mathbf{A})^{H} .
\end{aligned}
$$

Therefore, the optimum problem in (13) can be transformed into the following:

$$
\begin{array}{ll}
\min & \mathbf{w}^{H} \mathbf{w} \\
\text { s.t. } & \mathbf{w}^{H} \mathbf{P}_{(\mathbf{B}-\zeta \mathbf{A})}^{\perp} \mathbf{A}=1 \\
& \mathbf{B}-\zeta \mathbf{A} \in\langle\mathbf{w}\rangle^{\perp} .
\end{array}
$$

To solve this problem, a cost function is constructed by Lagrange multiplier method as

$$
F(w)=\mathbf{w}^{H} \mathbf{w}+\lambda\left(\mathbf{w}^{H} \mathbf{P}_{(\mathbf{B}-\zeta \mathbf{A})}^{\perp} \mathbf{A}-1\right) .
$$

Taking derivative of (18) with respect to $\mathbf{w}$, the optimum weight is obtained as

$$
\mathbf{w}=-\frac{\lambda}{2} \mathbf{P}_{(\mathbf{B}-\zeta \mathbf{A})}^{\perp} \mathbf{A}
$$

Substitute $w$ into the constraint condition $\mathbf{w}^{H} \mathbf{P}_{(\mathbf{B}-\zeta \mathbf{A})}^{\perp} \mathbf{A}=$ 1; we have

$$
-\frac{\lambda}{2} \mathbf{A}^{H} \mathbf{P}_{(\mathbf{B}-\zeta \mathbf{A})}^{\perp}{ }^{H} \mathbf{P}_{(\mathbf{B}-\zeta \mathbf{A})}^{\perp} \mathbf{A}=1 .
$$

Consider that $\mathbf{P}_{(\mathbf{B}-\zeta \mathbf{A})}^{\perp}{ }^{H}=\mathbf{P}_{(\mathbf{B}-\zeta \mathbf{A})}^{\perp}$ and $\mathbf{P}_{(\mathbf{B}-\zeta \mathbf{A})}^{\perp}{ }^{2}=\mathbf{P}_{(\mathbf{B}-\zeta \mathbf{A})}^{\perp}$; the cost factor is

$$
\lambda=-2\left(\mathbf{A}^{H} \mathbf{P}_{(\mathbf{B}-\zeta \mathbf{A})}^{\perp} \mathbf{A}\right)^{-1} .
$$

Substitute $\lambda$ into (19); we have the explicit weight as

$$
\begin{aligned}
\mathbf{w} & =\mathbf{P}_{(\mathbf{B}-\zeta \mathbf{A})}^{\perp} \mathbf{A}\left(\mathbf{A}^{H} \mathbf{P}_{(\mathbf{B}-\zeta \mathbf{A})}^{\perp} \mathbf{A}\right)^{-1} \\
& =\left(\left(\mathbf{A}^{H} \mathbf{P}_{(\mathbf{B}-\zeta \mathbf{A})}^{\perp} \mathbf{A}\right)^{-1} \mathbf{A}^{H} \mathbf{P}_{(\mathbf{B}-\zeta \mathbf{A})}^{\perp}\right)^{H} \\
& =\left(\frac{\mathbf{A}^{H}}{\|\mathbf{A}\|^{2}} \mathbf{E}_{\mathbf{A}, \mathbf{B}, \zeta}\right)^{H},
\end{aligned}
$$

where the GOP operator is derived from (22) as

$$
\mathbf{E}_{\mathbf{A}, \mathbf{B}, \zeta}=\mathbf{A}\left(\mathbf{A}^{H} \mathbf{P}_{(\mathbf{B}-\zeta \mathbf{A})}^{\perp} \mathbf{A}\right)^{-1} \mathbf{A}^{H} \mathbf{P}_{(\mathbf{B}-\zeta \mathbf{A})}^{\perp} .
$$

Compare (23) with (5); the expressions of GOP and OP share the similar expression. It will be shown that the GOP operator presented is related to the $\mathrm{OP}$ operator and orthogonal projection operator. However, GOP is the extended form of them and some special properties are generated by the GOP. 
3.2. Properties of the GOP. Four fundamental properties of the GOP are presented. The idempotence of GOP and the oblique projection abilities are given, respectively, in Property 1 . The connections of OP and orthogonal projection with GOP are shown in Property 2. The enlargement of the principle angle by GOP compared to OP is discussed in Property 3, which implies a reduction of the noise power output. Lastly, in Property 4, the maximized SINR condition in GOP is presented.

Property 1. Consider

$$
\begin{gathered}
\mathbf{E}_{\mathbf{A}, \mathbf{B}, \zeta}^{2}=\mathbf{E}_{\mathbf{A}, \mathbf{B}, \zeta}, \\
\mathbf{E}_{\mathbf{A}, \mathbf{B}, \zeta} \mathbf{A}=\mathbf{A}, \\
\mathbf{E}_{\mathbf{A}, \mathbf{B}, \zeta} \mathbf{B}=\zeta \mathbf{A} .
\end{gathered}
$$

Examine (24); the GOP operator has equivalent filtering effect when the operator is multiplied. It recovers the desired signal without amplitude and phase distortion, whereas part of the interference is projected into the target subspace and is reserved by GOP. The target signal after GOP filtering can be expressed as

$$
\begin{aligned}
\widehat{S}_{\mathrm{GOP}}(t) & =\frac{\mathbf{A}^{H}}{\|\mathbf{A}\|^{2}} \mathbf{E}_{\mathbf{A}, \mathbf{B}, \zeta} \mathbf{x}(t) \\
& =s(t)+\zeta i(t)+\frac{\mathbf{A}^{H}}{\|\mathbf{A}\|^{2}} \mathbf{E}_{\mathbf{A}, \mathbf{B}, \zeta} \mathbf{n}(t) .
\end{aligned}
$$

Proof. Since $\mathbf{P}_{(\mathbf{B}-\zeta \mathbf{A})}^{\perp}$ is a square matrix and $\mathbf{A}$ is a column vector, we have

$$
\begin{aligned}
& \mathbf{E}_{\mathbf{A}, \mathbf{B}, \zeta}^{2}= \mathbf{A}\left(\mathbf{A}^{H} \mathbf{P}_{(\mathbf{B}-\zeta \mathbf{A})}^{\perp} \mathbf{A}\right)^{-1} \mathbf{A}^{H} \mathbf{P}_{(\mathbf{B}-\zeta \mathbf{A})}^{\perp} \\
& \cdot \mathbf{A}\left(\mathbf{A}^{H} \mathbf{P}_{(\mathbf{B}-\zeta \mathbf{A})}^{\perp} \mathbf{A}\right)^{-1} \mathbf{A}^{H} \mathbf{P}_{(\mathbf{B}-\zeta \mathbf{A})}^{\perp} \\
&= \mathbf{A}\left(\mathbf{A}^{H} \mathbf{P}_{(\mathbf{B}-\zeta \mathbf{A})}^{\perp} \mathbf{A}\right)^{-1}\left(\mathbf{A}^{H} \mathbf{P}_{(\mathbf{B}-\zeta \mathbf{A})}^{\perp} \mathbf{A}\right) \\
& \times\left(\mathbf{A}^{H} \mathbf{P}_{(\mathbf{B}-\zeta \mathbf{A})}^{\perp} \mathbf{A}\right)^{-1} \mathbf{A}^{H} \mathbf{P}_{(\mathbf{B}-\zeta \mathbf{A})}^{\perp} \\
&= \mathbf{A}\left(\mathbf{A}^{H} \mathbf{P}_{(\mathbf{B}-\zeta \mathbf{A})}^{\perp} \mathbf{A}\right)^{-1} \mathbf{A}^{H} \mathbf{P}_{(\mathbf{B}-\zeta \mathbf{A})}^{\perp}=\mathbf{E}_{\mathbf{A}, \mathbf{B}, \zeta}, \\
& \mathbf{E}_{\mathbf{A}, \mathbf{B}, \zeta} \mathbf{A}= \mathbf{A}\left(\mathbf{A}^{H} \mathbf{P}_{(\mathbf{B}-\zeta \mathbf{A})}^{\perp} \mathbf{A}\right)^{-1}\left(\mathbf{A}^{H} \mathbf{P}_{(\mathbf{B}-\zeta \mathbf{A})}^{\perp} \mathbf{A}\right)=\mathbf{A} . \\
& \text { Since } \mathbf{B}-\zeta \mathbf{A} \text { is a column vector, from }(16) \text { we have } \\
& \mathbf{P}_{(\mathbf{B}-\zeta \mathbf{A})}^{\perp}(\mathbf{B}-\zeta \mathbf{A})=0, \\
& \mathbf{E}_{\mathbf{A}, \mathbf{B}, \zeta}(\mathbf{B}-\zeta \mathbf{A})= \mathbf{A}\left(\mathbf{A}^{H} \mathbf{P}_{(\mathbf{B}-\zeta \mathbf{A})}^{\perp} \mathbf{A}\right)^{-1} \mathbf{A}^{H} \mathbf{P}_{(\mathbf{B}-\zeta \mathbf{A})}^{\perp}(\mathbf{B}-\zeta \mathbf{A}) \\
&= 0=\mathbf{E}_{\mathbf{A}, \mathbf{B}, \zeta} \mathbf{B}=\zeta \mathbf{A} .
\end{aligned}
$$

Property 2. The ISC $\zeta$ can be real or complex, and its absolute value $|\zeta| \in[0,1]$. When $\zeta=\langle\mathbf{A}, \mathbf{B}\rangle /\|\mathbf{A}\|\|\mathbf{B}\|$, GOP becomes an orthogonal operator, and

$$
\mathbf{E}_{\mathbf{A}, \mathbf{B}, \zeta}=\mathbf{P}_{\mathbf{A}}, \quad \zeta=\frac{\langle\mathbf{A}, \mathbf{B}\rangle}{\|\mathbf{A}\|\|\mathbf{B}\|}
$$

When $\zeta=0$, GOP degrades to an OP and shares the same expression in (5), and

$$
\mathbf{E}_{\mathbf{A}, \mathbf{B}, \zeta}=\mathbf{E}_{\mathbf{A}, \mathbf{B}}, \quad \zeta=0 .
$$

Proof. Suppose $\zeta$ equals the inner product of $\mathbf{A}$ and $\mathbf{B}$;

$$
\begin{aligned}
\langle\mathbf{A},(\mathbf{B}-\zeta \mathbf{A})\rangle & =\mathbf{A}^{H}(\mathbf{B}-\zeta \mathbf{A}) \\
& =\mathbf{A}^{H} \mathbf{B}-\frac{\mathbf{A}^{H} \mathbf{A} \mathbf{A}^{H} \mathbf{B}}{\|\mathbf{A}\|\|\mathbf{B}\|}=0 .
\end{aligned}
$$

Therefore, subspace $\langle\mathbf{A}\rangle$ is orthogonal to subspace $\langle\mathbf{B}-$ $\zeta \mathbf{A}\rangle$, and substitute (16) into (23); we have

$$
\mathbf{E}_{\mathbf{A}, \mathbf{B}, \zeta}=\left(\mathbf{E}_{\mathbf{A}, \mathbf{B}, \zeta}\right)^{H}=\mathbf{A}\left(\mathbf{A}^{H} \mathbf{A}\right)^{-1} \mathbf{A}^{H}=\mathbf{P}_{\mathbf{A}},
$$

where $\mathbf{P}_{\mathbf{A}}$ represents the orthogonal projection of $\mathbf{A}$.

Suppose $\zeta$ is set to zero; hence

$$
\begin{aligned}
\mathbf{E}_{\mathbf{A}, \mathbf{B}, \zeta} & =\mathbf{A}\left(\mathbf{A}^{H} \mathbf{P}_{(\mathbf{B}-\zeta \mathbf{A})}^{\perp} \mathbf{A}\right)^{-1} \mathbf{A}^{H} \mathbf{P}_{(\mathbf{B}-\zeta \mathbf{A})}^{\perp} \\
& =\mathbf{A}\left(\mathbf{A}^{H} \mathbf{P}_{\mathbf{B}}^{\perp} \mathbf{A}\right)^{-1} \mathbf{A}^{H} \mathbf{P}_{\mathbf{B}}^{\perp}=\mathbf{E}_{\mathbf{A}, \mathbf{B}} .
\end{aligned}
$$

Correspondingly, the target signal recovered by GOP and $\mathrm{OP}$ is identical in this case:

$$
\widehat{S}_{\mathrm{GOP}}(t)=\widehat{S}_{\mathrm{OP}}(t), \quad \zeta=0 .
$$

Property 3. The principle angle of GOP is adjusted by $\zeta$. When $\zeta \in(0, \cos \psi]$, the principle angle of GOP can be enlarged, which is given by

$$
\psi^{\prime}>\psi, \quad \zeta \in(0, \cos \psi]
$$

where $\psi^{\prime}$ represents the principle angle of GOP and $\psi$ represents the principle angle of OP under the same $\mathbf{A}$ and $\mathbf{B}$. With a proper setting of the ISC, the principle angle between subspaces can be enlarged. 
Proof. The square of the cosine of the new principle angle is

$$
\begin{aligned}
\cos ^{2} \psi^{\prime} & =\frac{\left(\mathbf{A}^{H} \mathbf{B}^{\prime}\right)^{H}\left(\mathbf{A}^{H} \mathbf{B}^{\prime}\right)}{\left\|\mathbf{A}^{H}\right\|^{2}\left\|\mathbf{B}^{\prime}\right\|^{2}} \\
& =\frac{(\mathbf{B}-\zeta \mathbf{A})^{H} \mathbf{A} \mathbf{A}^{H}(\mathbf{B}-\zeta \mathbf{A})}{\mathbf{A}^{H} \mathbf{A}(\mathbf{B}-\zeta \mathbf{A})^{H}(\mathbf{B}-\zeta \mathbf{A})} \\
& =\frac{\left(\mathbf{B}^{H} \mathbf{A}^{H} \mathbf{A} \mathbf{B} / \mathbf{A}^{H} \mathbf{A}\right)-\zeta\left(\mathbf{A}^{H} \mathbf{B}+\mathbf{B}^{H} \mathbf{A}\right)+\zeta^{2} \mathbf{A}^{H} \mathbf{A}}{\mathbf{B}^{H} \mathbf{B}-\zeta\left(\mathbf{A}^{H} \mathbf{B}+\mathbf{B}^{H} \mathbf{A}\right)+\zeta^{2} \mathbf{A}^{H} \mathbf{A}} \\
& =\frac{(\cos \psi-\zeta)^{2}}{\zeta^{2}-2 \zeta \cos \psi+1} \\
& =\frac{1}{1+\left(1-\cos { }^{2} \psi\right) /(\cos \psi-\zeta)^{2}} .
\end{aligned}
$$

Since (35) is a monotonically declining function of $\zeta$ under the condition of $\zeta \in[0, \cos \psi]$, it is evident that

$$
\begin{gathered}
\psi^{\prime}=\psi, \quad \zeta=0, \\
\text { or } \psi^{\prime}>\psi, \quad \zeta \in(0, \cos \psi] .
\end{gathered}
$$

Equation (37) demonstrates that when $\zeta$ is set in the range of $(0, \cos \psi]$, the principle angle of GOP is enlarged. According to the expression of output noise power in (12), the noise power of GOP will be less than that of OP:

$$
P_{n}^{\prime}<P_{n}
$$

Property 4. Suppose INR is known a priori; a maximized SINR, which is the SINR upper bound of GOP, can be obtained and the corresponding ICS is given as

$$
\zeta_{\text {opt }}=\frac{\cos \psi}{1+N \cdot \operatorname{INR} \sin ^{2} \psi}
$$

such that the maximum SINR is obtained as

$$
\mathrm{SINR}_{\text {max }}=\frac{\left(\mathrm{SIR}+N \cdot \mathrm{SNR} \sin ^{2} \psi\right) N \cdot \mathrm{SNR}}{\mathrm{SIR}+N \cdot \mathrm{SNR}},
$$

where $N$ represents the number of array elements.

Proof. The SINR output of the GOP filter can be expressed as

$$
\begin{aligned}
\text { SINR } & =\frac{P_{s}}{P_{i}^{\prime}+P_{n}^{\prime}}=\frac{P_{s}}{\zeta^{2} P_{i}+P_{n} / N \sin ^{2} \psi^{\prime}} \\
& =\left(\frac{\zeta^{2}}{\operatorname{SIR}}+\frac{1}{N \sin ^{2} \psi^{\prime} \mathrm{SNR}}\right)^{-1} \\
& =\left(\frac{\zeta^{2}}{\operatorname{SIR}}+\frac{1-2 \zeta \cos \psi+\zeta^{2}}{N \sin ^{2} \psi \mathrm{SNR}}\right)^{-1} .
\end{aligned}
$$

To have the maximum value of SINR, the first order derivative of (41) with respect to $\zeta$ is taken; therefore the optimum $\zeta_{\text {opt }}$ that will give the maximum SINR in (40) is obtained as in (39).

\section{GOP in Spatial Filtering}

By using the spatial difference between the target and interference, spatial filtering is an effective method to reduce the power of unwanted signals including output noise. In the construction of spatial GOP filter, the design of ISC parameter is flexible. It can be generated by using the conventional adaptive spatial filtering methods such as minimum variance distortionless response (MVDR) algorithm [20].

The constrained criterion of MVDR is described as

$$
\begin{array}{ll}
\min _{\mathbf{w}} & \mathbf{w}^{T} \mathbf{R w} \\
\text { s.t. } & \mathbf{w}^{H} \mathbf{A}=1,
\end{array}
$$

where $\mathbf{A}$ is the spatial vector for the target signal and $\mathbf{R}=$ $E\left\{\mathbf{x}(t) \mathbf{x}^{H}(t)\right\}$ represents the covariance matrix of the received signal.

The weight of MVDR can be obtained as

$$
\mathbf{w}_{\mathrm{MVDR}}=\mathbf{R}^{-1} \mathbf{A}\left(\mathbf{A}^{H} \mathbf{R}^{-1} \mathbf{A}\right)^{-1} .
$$

Therefore, the ISC obtained from $\mathbf{w}_{\text {MVDR }}$ is expressed as

$$
\zeta_{\mathrm{MVDR}}=\mathbf{w}_{\mathrm{MVDR}}^{H} \mathbf{B}=\left(\mathbf{R}^{-1} \mathbf{A}\left(\mathbf{A}^{H} \mathbf{R}^{-1} \mathbf{A}\right)^{-1}\right)^{H} \mathbf{B} .
$$

It should be noted that the restoration of the target is not related to $\zeta$, which is the fundamental property of GOP as given in (25). The interference suppression effect is determined by $\zeta$ only. As a result, except for MVDR, more adaptive beamforming methods can be utilized. However, as the information required by MVDR is usually easy to obtain, it is more representative to derive $\zeta$ from $\mathbf{w}_{\text {MVDR }}$. In the following part, the SINR performance of the MVDR, OP, and GOP with $\zeta$ given in (44) is analyzed and compared.

4.1. SINRs Comparison of GOP and MVDR. The SINR performance of the GOP and MVDR filter is evaluated. The SINRs for GOP and MVDR filter are given in (45) and (46), respectively, as

$$
\begin{aligned}
\operatorname{SINR}_{\mathrm{GOP}} & =\frac{E\left\{|\mathbf{w A} s(n)|^{2}\right\}}{E\left\{|\mathbf{w B} i(n)|^{2}\right\}+E\left\{|\mathbf{w n}(n)|^{2}\right\}} \\
& =\frac{E\left\{|\mathbf{w} \mathbf{A}|^{2}\right\} \sigma_{s}^{2}}{E\left\{|\mathbf{w B}|^{2}\right\} \sigma_{i}^{2}+|\mathbf{w}|^{2} \sigma_{n}^{2}},
\end{aligned}
$$

where $\mathbf{w}=\mathbf{A}^{H} \mathbf{E}_{\mathbf{A}, \mathbf{B}, \zeta} /\|\mathbf{A}\|^{2}$. Consider

$$
\begin{aligned}
\operatorname{SINR}_{\mathrm{MVDR}} & =\frac{E\left\{\left|\mathbf{w}_{\mathrm{MVDR}} \mathbf{A} s(n)\right|^{2}\right\}}{E\left\{\left|\mathbf{w}_{\mathrm{MVDR}} \mathbf{B} i(n)\right|^{2}\right\}+E\left\{\left|\mathbf{w}_{\mathrm{MVDR}} \mathbf{n}(n)\right|^{2}\right\}} \\
& =\frac{E\left\{\left|\mathbf{w}_{\mathrm{MVDR}} \mathbf{A}\right|^{2}\right\} \sigma_{s}^{2}}{E\left\{\left|\mathbf{w}_{\mathrm{MVDR}} \mathbf{B}\right|^{2}\right\} \sigma_{i}^{2}+\left|\mathbf{w}_{\mathrm{MVDR}}\right|^{2} \sigma_{n}^{2}} .
\end{aligned}
$$


Observing (13), (42), (43), and (44), we get

$$
\begin{aligned}
& \mathbf{w}^{H} \mathbf{A}=\mathbf{w}_{\mathrm{MVDR}}^{H} \mathbf{A}, \\
& \mathbf{w}^{H} \mathbf{B}=\mathbf{w}_{\mathrm{MVDR}}^{H} \mathbf{B} .
\end{aligned}
$$

Substitute (47) into (45) and (46); it can be observed that the difference of output SINRs is decided by the power of noise after filtering.

Investigating the objective function in (13), we get $\mathbf{w}_{\mathrm{GOP}}$ which satisfies $\min \left(\mathbf{w}_{\mathrm{GOP}}^{H} \mathbf{w}_{\mathrm{GOP}}\right)$; hence $\left|\mathbf{w}_{\mathrm{GOP}}\right|^{2} \leq\left|\mathbf{w}_{\mathrm{MVDR}}\right|^{2}$. This leads to

$$
\operatorname{SINR}_{\mathrm{GOP}} \geq \operatorname{SINR}_{\mathrm{MVDR}} \text {. }
$$

Both of these filters attempt to reduce the interference and noise while producing the distortionless response. However, the GOP filter has a better treatment to the noise and interference which results in a higher SINR.

4.2. SINRs Comparison of GOP and OP. The SINR performance of the GOP and OP filter is evaluated. The SINR for OP filter is written as

$$
\begin{aligned}
\operatorname{SINR}_{\mathrm{OP}} & =\frac{E\left\{\left|\mathbf{w}_{\mathrm{OP}} \mathbf{A} s(n)\right|^{2}\right\}}{E\left\{\left|\mathbf{w}_{\mathrm{OP}} \mathbf{B} i(n)\right|^{2}\right\}+E\left\{\left|\mathbf{w}_{\mathrm{OP}} \mathbf{n}(n)\right|^{2}\right\}} \\
& =\frac{\sigma_{s}^{2}}{\sigma_{n}^{2}\left\|\mathbf{E}_{\mathrm{AB}}\right\|^{2} /\|\mathbf{A}\|^{2}},
\end{aligned}
$$

where $\mathbf{w}_{\mathrm{OP}}=\mathbf{A}^{H} \mathbf{E}_{\mathbf{A}, \mathbf{B}} /\|\mathbf{A}\|^{2}$.

Let the ratio between SINRs of GOP and OP filter be

$$
\begin{aligned}
k & =\frac{\operatorname{SINR}_{\mathrm{GOP}}}{\operatorname{SINR}_{\mathrm{OP}}} \\
& =\frac{\left\|\mathbf{E}_{\mathbf{A B}}\right\|^{2} \sigma_{n}^{2} /\|\mathbf{A}\|^{2}}{\zeta^{2} \sigma_{i}^{2}+\left|\mathbf{A}^{H} \mathbf{E}_{\mathbf{A}, \mathbf{B}, \zeta} /\|\mathbf{A}\|^{2}\right|^{2} \sigma_{n}^{2}} \\
& =\frac{1}{N \zeta^{2} \mathrm{INR} \sin ^{2} \psi+\|\mathbf{B}-\zeta \mathbf{A}\|^{2} / N} \\
& =\frac{1}{Z \zeta^{2}-2 \cos \psi \zeta+1},
\end{aligned}
$$

where $Z=1+N \sin ^{2} \psi \mathrm{INR},\|\mathbf{A}\|^{2}=N$, and $N$ is the number of the array elements.

As the value of $k$ is determined by the principle angle, INR, the number of array elements, and ISC simultaneously, it is not easy to be analyzed without numerical evaluations. However, we can make a reasonable assumption under two extreme cases, where spatial difference between the target and interference approaches 90 degrees or 0 degrees.

Case $1\left(\psi \rightarrow 90^{\circ}\right)$. The angular separation between target and interference is nearly orthogonal, which means the spatial difference is maximized. An aggressive action is taken, and the interference is expected to be eliminated completely. Rewrite (50) as

$$
k_{1}=\frac{1}{N \sin ^{2} \psi \varepsilon_{1}+2 \delta_{1}+1+\zeta^{2}},
$$

where $\varepsilon_{1}=\zeta^{2}$ INR and $\delta_{1}=\zeta \cos \psi$.

To evaluate (51) numerically, let $\psi=89.8^{\circ}$ and $\zeta=0$; we have $\sin ^{2} \psi \approx 1, \zeta^{2}=0$, and $\delta_{1}=0$. Since the INR is bounded by a practical maximum of $60 \mathrm{~dB}$, we have $\varepsilon_{1}=0, k_{1}=1$, and

$$
\operatorname{SINR}_{\mathrm{GOP}}=\operatorname{SINR}_{\mathrm{OP}} \text {. }
$$

Case $2\left(\psi \rightarrow 0^{\circ}\right)$. When the angular separation between target and interference is very close, the principle angle is near zero. For OP filter, its SINR is not satisfying due to the amplified noise power as given in (12). However, the proposed GOP filter can be expected to be more conservative in rejecting the interference and shifting the focus in reducing the noise power output. That means when INR is low, which means noise power is comparably high, the ISC is approaching one. However, if noise power is comparably much lower than interference, resulting in a very high INR, the ISC is approaching zero to facilitate interference suppression. We rewrite (50) as

$$
k_{2}=\frac{1}{N \varepsilon_{2}+\delta_{2}+\zeta^{2}},
$$

where $\varepsilon_{2}=(\zeta \sin \psi)^{2}$ INR and $\delta_{1}=2 \zeta \cos \psi+1$.

To evaluate (53) numerically, let $\psi=0.01^{\circ}$ and $\zeta \epsilon$ $\left[10^{-5}, 1\right]$; we have $\sin ^{2} \psi=0$; hence $\varepsilon_{2}=0$. Therefore, we have $\delta_{2}+\zeta^{2} \geq 1, k_{2} \geq 1$, and

$$
\operatorname{SINR}_{\mathrm{GOP}} \geq \operatorname{SINR}_{\mathrm{OP}}
$$

From the theoretical analysis of the two cases, the SINR outputs from both filters have an identical performance when the spatial difference between the target and interference is large. However, GOP filter has a superior performance than OP filter when the spatial difference is small.

Moreover, according to Property 4 of GOP, there is an optimized $\zeta_{\text {opt }}$ in maximizing SINR output if the INR can be obtained in advance. By substituting (39), (50) can be rewritten as

$$
\begin{aligned}
k & =\frac{1}{Z\left(\zeta_{\text {opt }}-\cos \psi / Z\right)^{2}+1-\cos ^{2} \psi / Z} \\
& =\frac{1}{1-\cos ^{2} \psi / Z} \geq 1,
\end{aligned}
$$

where $\zeta_{\text {opt }}=\cos \psi / Z$.

Therefore, we have

$$
\operatorname{SINR}_{\mathrm{GOP}} \geq \operatorname{SINR}_{\mathrm{OP}}
$$

4.3. Simulations. Assume an ULA of 10 antenna elements with half wavelength spacing between adjacent elements; the carrier frequency is $f_{0}=5 \mathrm{MHz}$. Under the conditions of 


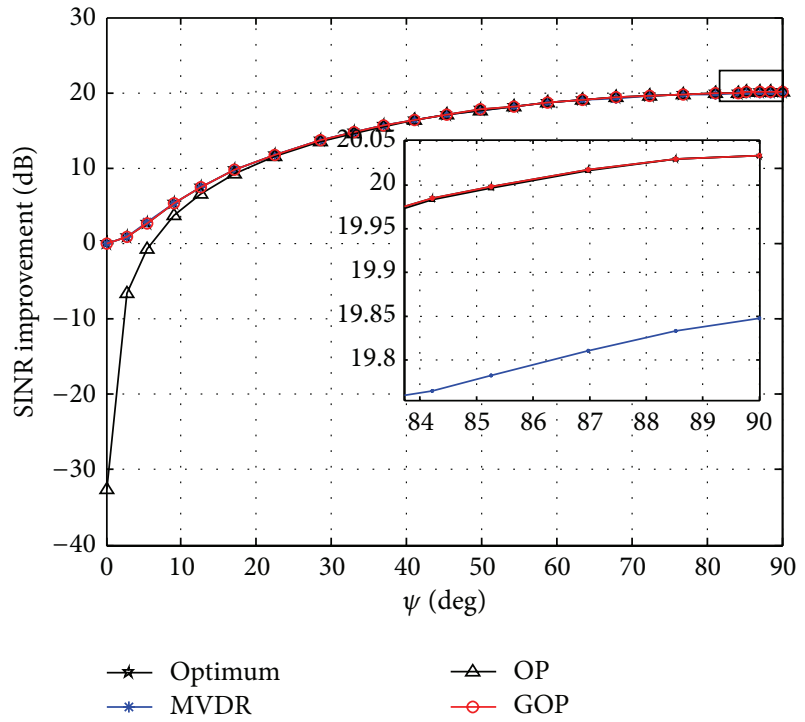

FIgURE 1: SINR improvement versus $\psi$.

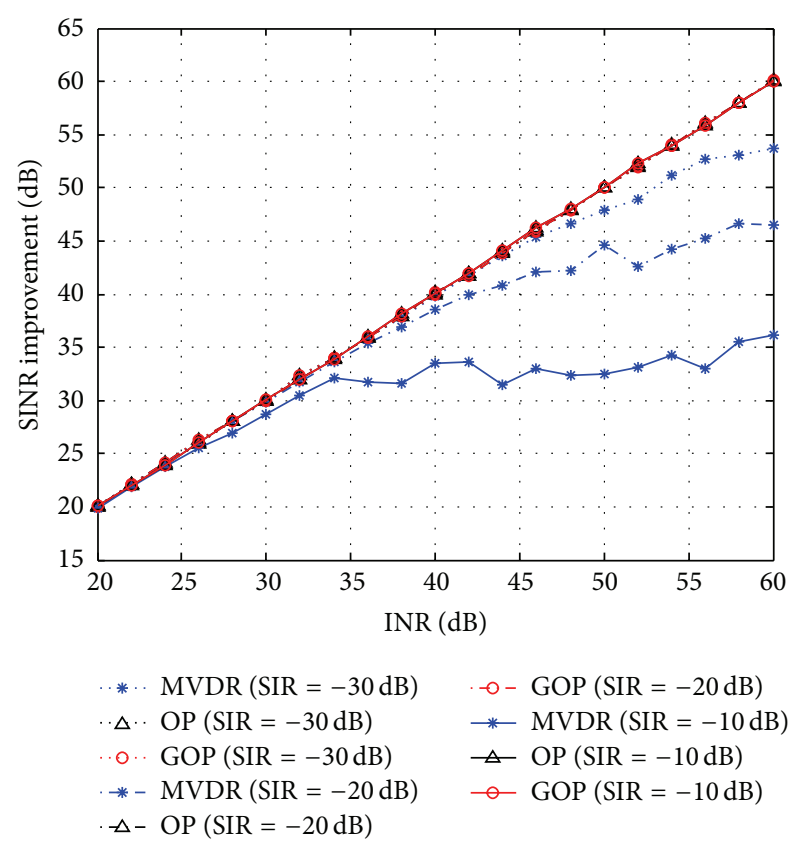

FIGURE 2: SINR improvement versus INR for Case 1.

SNR $=10 \mathrm{~dB}$ and SIR $=-10 \mathrm{~dB}$, the SINR improvement versus $\psi$ for MVDR, OP, GOP, and GOP with optimum ISC is shown in Figure 1.

From Figure 1, it can be observed that the principle angles have great impacts on the SINR performance of the three spatial filters. With the principle angle less than 10 degrees, a sharper decrease appears in SINR for the OP filter than both GOP and MVDR filters. However, when the principle angle increases to nearly 90 degrees, GOP and OP filters perform slightly better than MVDR filter. The GOP spatial filter demonstrates a superior performance than $\mathrm{OP}$ and

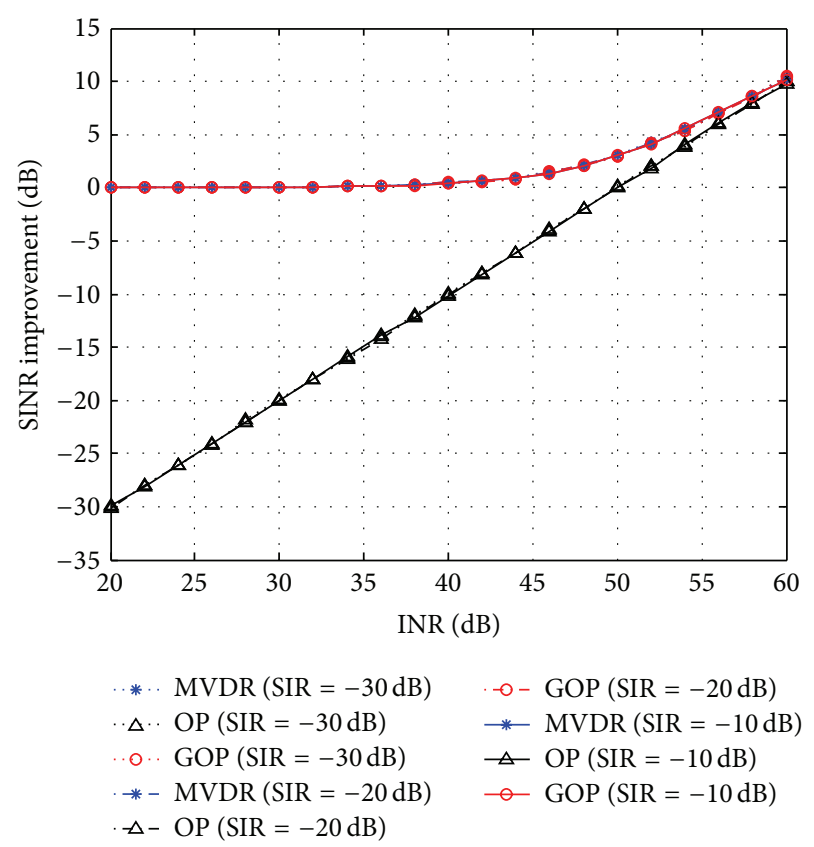

FIGURE 3: SINR improvement versus INR for Case 2.

MVDR filters in the whole angle range; further it coincides with the maximized SINR derived in (40).

In the following, the principle angle is fixed and the performances of three filters under different INRs are investigated. The INRs span from $20 \mathrm{~dB}$ to $60 \mathrm{~dB}$ while the SIRs are of $-10 \mathrm{~dB},-20 \mathrm{~dB}$, and $-30 \mathrm{~dB}$, respectively. The principle angles are set to near 90 degrees and 0 degrees, respectively, which correspond to the cases in Section 4.2.

In Figures 2 and 3, the principle angles are set to 89.8 degrees and 0.18 degrees, respectively, which correspond to Cases 1 and 2. It is found that the ISC varies slightly around zero when the INRs change for Case 1, and ISC varies in range of $\left[2.7 \times 10^{-5}, 1\right]$ for Case 2 . As shown in Figure 2, the SINR improvements of the GOP and OP increase linearly with INRs; however, the MVDR shows an inferior SINR improvement. In Figure 3, the principle angle is small, which means that the spatial difference between the target and interference reduces. The noise power amplifying effect of OP is significant, resulting in a severe SINR performance loss. In contrast, the GOP and MVDR filters have better SINR improvements, which exceed $30 \mathrm{~dB}$ at highest than OP filter. In general, the GOP filter generates a superior performance than both OP and MVDR filters. The numerical simulations have verified the theoretical analysis of the spatial filters presented.

\section{GOP in Polarization Filtering of HF Radar}

High frequency surface wave radar (HFSWR) [22] operates in the short wave band, which facilities the over-the-horizon detection over the sea surface. However, signals in this band will also get reflected by the ionosphere, leading to a strong interference to the target detection or sea state remote sensing. In this section, we explored the polarization information 
in the HFSWR and extended the application of the GOP filter in polarization filtering; further, a challenging ionospheric clutter mitigation problem in HFSWR is addressed.

5.1. Generalized Oblique Projection Polarization Filter. As the linear signal model presented in (4), a pair of the polarization parameters represent the amplitude relationship and the phase difference between the echoes received by the orthogonal dual-polarized radar sensor.

Suppose the polarization parameters of the target and interference are different; then the target polarization subspace $\left\langle\mathbf{P}_{\mathbf{s}}\right\rangle$ and interference polarization subspace $\left\langle\mathbf{P}_{\mathbf{i}}\right\rangle$ are disjoint. Note that the polarization subspace is independent of the specific radar signal waveforms. The polarization subspace is constructed by the difference of the polarization state. Therefore, the target and interference subspaces in (23) can be given as

$$
\mathbf{A}=\left[\begin{array}{c}
\cos \gamma_{s} \\
\sin \gamma_{s} e^{j \eta_{s}}
\end{array}\right], \quad \mathbf{B}=\left[\begin{array}{c}
\cos \gamma_{i} \\
\sin \gamma_{i} e^{j \eta_{i}}
\end{array}\right],
$$

where $\gamma$ and $\eta$ are the polarization pair with subscripts $s$ and $i$ representing target and interference.

There are two sets of the parameters that need to be decided, that is, the echoes polarization set and the ISC. The polarization angle is obtained as

$$
\gamma=\operatorname{tg}^{-1}\left(\frac{\left|x_{V}(t)\right|}{\left|x_{H}(t)\right|}\right)
$$

and the polarization phase difference as

$$
\eta=\arg \left(x_{V}(t)\right)-\arg \left(x_{H}(t)\right),
$$

where $x_{V}(t), x_{H}(t)$ represent the vertically and horizontally polarized signal components and $\arg (\cdots)$ indicates phase of the signal.

By the inflexibility of the polarized state in the temporal domain and the frequency domain [18], these parameters can be calculated using frequency transformed signal also. The ISC is a free variable, which can be designed according to the specific application.

However, it can be proved that if the ISC is set to zero, this GOP polarization filter becomes OP polarization filter. In constrained condition, that is, subspace $\left\langle\mathbf{P}_{\mathbf{s}}\right\rangle$ is orthogonal to $\left\langle\mathbf{P}_{\mathbf{i}}\right\rangle$, the GOP polarization filter degrades into an orthogonal polarization filter.

5.2. Ionospheric Clutter Cancellation in HFSWR. The characteristics of the ionosphere reflected echoes received by HFSWR are

(1) nonstationary,

(2) multiclutters,

(3) widely spread in range and Doppler bins.

In addition to that, there are ionospheric clutters returning from the sea surface, which make their polarization states close to the target, or the spatial difference between the

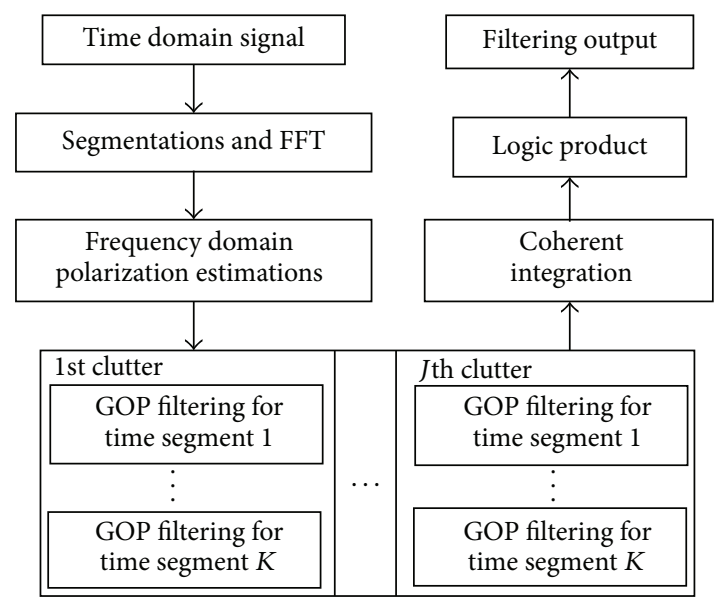

FIGURE 4: Ionospheric clutter mitigation process flow.

clutters and target is not obvious. Those characteristics lead to an unsolved clutter mitigation problem so far.

As the radar arrays cannot effectively distinguish the target and interference spatially in this scenario, the array polarization diversity and the coherent integration process are explored. To handle those characteristics, segmentations of the time domain signals are introduced in an effort to approach the real clutter parameters, and multiple GOP filters with flexible parameters $\zeta$ are constructed. The flowchart to illustrate the ionospheric clutter mitigation process is shown in Figure 4.

In the dual-polarized HFSWR system, the time domain signal is range processed. After proper signal segmentations, the polarization states are estimated according to (58) and (59) with frequency transformed signal components. The signals returned from the ship target over sea surface will retain vertically polarized state, whereas the ionosphere reflected clutters will be ellipse polarized with strong horizontally polarized components. The target and clutter polarization subspaces (A and $\mathbf{B}$ for GOP) are therefore obtained by the polarization threshold. A total number of $K$ segments of the time divided data after filtering are connected and coherent integration is performed. However, by the special design of GOP polarization filter, an extra integration loss to the ionospheric clutter will be brought in.

By Property 1 of GOP, the target polarization subspaces are retained, while the ionospheric clutters are controlled by the free variable $\zeta$ which is designed to introduce random phase shift. Therefore, after GOP processing, a significant coherent integration loss will be introduced to the ionospheric clutters when the time divided segments are reconnected and fast Fourier transformed. However, the target's amplitude and phase are unchanged and its coherent integration performance is not affected.

The free variable of $\zeta(n)$ is designed as

$$
\zeta(n)=\operatorname{rnd}\left(\delta_{1}, \delta_{2}, n\right) e^{\operatorname{jrnd}\left(\beta_{1}, \beta_{2}, n\right)}, \quad n \in[1, K],
$$

where $\operatorname{rnd}\left(\delta_{1}, \delta_{2}, n\right)$ represents random arrays from the uniform distribution with amplitude range $\left[\delta_{1}, \delta_{2}\right]$ and random 


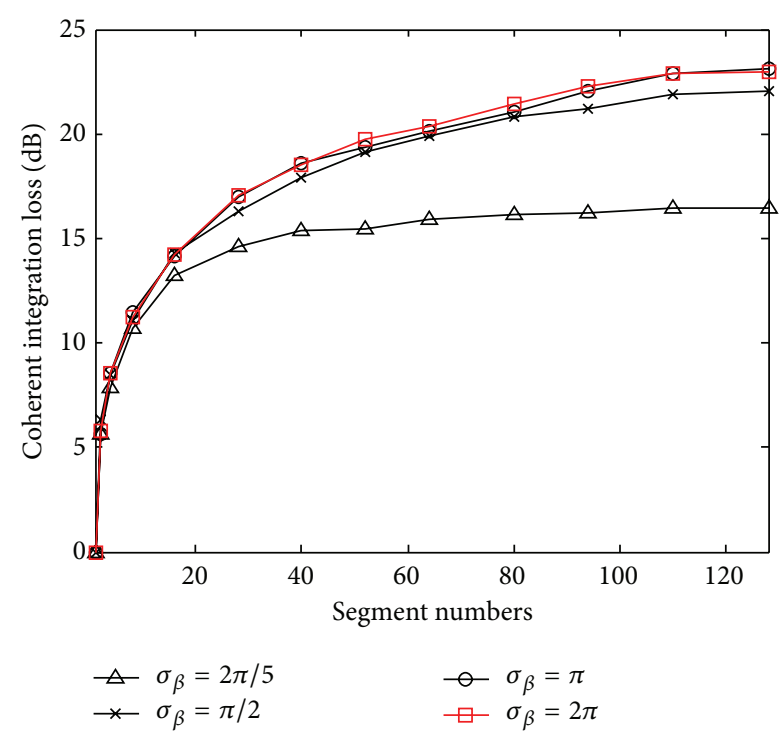

Figure 5: Coherent loss versus segment numbers under different phase ranges.

phase term $e^{j \operatorname{rnd}\left(\beta_{1}, \beta_{2}, n\right)}$ with phase range $\left[\beta_{1}, \beta_{2}\right]$ and $n$ denotes the sequence number of $\zeta$ generated.

In deciding the parameters of $\zeta$, the number of segmentation and the phase ranges are of importance. In Figure 5, the segmentation number is chosen to be 64 and at that point phase range of $[0,2 \pi]$ is picked by simulation results of ideal exponential signals. The reasons are that more segmentation numbers can only introduce at most $3 \mathrm{~dB}$ loss further, while more segments indicate shorter time in coherent integration and result in more estimation errors, which may degrade the performance of the filter. Therefore, the parameters are obtained in consideration of the extra coherent loss and potential estimation error.

After logic product process [18], which reserves the smallest points in Doppler spectrum from all the filtering results, the final output in each range bin is obtained as

$$
\begin{aligned}
\mathbf{Y}(\omega)=\sum_{n=1}^{N}\left\{\delta\left(\omega-\omega_{n}\right)\right. \\
\left.\quad \times \min _{j=1}^{J}\left(\operatorname{FFT}\left[\sum_{k=1}^{K} \mathbf{E}_{\mathbf{A}, \mathbf{B}, \xi, k, j} \mathbf{x}_{k}\left(n_{\text {seg }, k}\right)\right]\right)\right\},
\end{aligned}
$$

where $N$ represents the length of FFT, $\omega_{n}$ represents the frequency at point $n, J$ represents the total number of the clutters, $K$ is the total number of segmentations, $\mathbf{E}_{\mathbf{A}, \mathbf{B}, \xi, k, j}$ is the $k$ th GOP in time domain for the suppression of $j$ th clutter, and $\mathbf{x}_{k}\left(n_{\text {seg, }, k}\right)$ is the $k$ th time segmented data with $n_{\text {seg, } k}$ denoting the sampled points in the corresponding segment.

In the HFSWR field experiment, the vertically polarized signal was transmitted with a carrier frequency of $7 \mathrm{Mhz}$ by a half-wave dipole antenna. The data was collected by a distant polarized high frequency linear array with 15-meter separation between the vertically and horizontally polarized elements. The coherent integration time was 4 minutes.

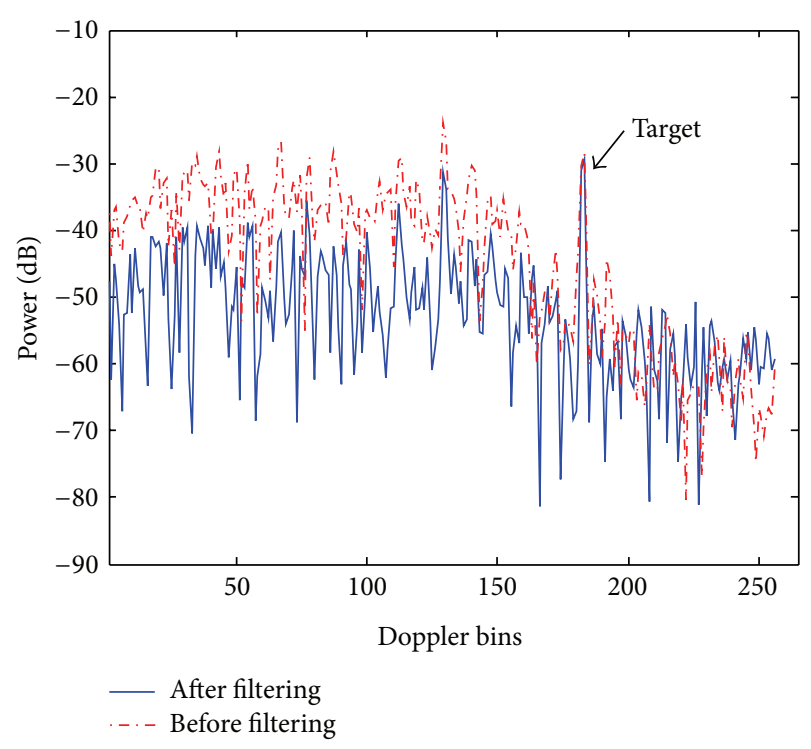

Figure 6: Doppler spectrum at range bin 79.

Figure 6 gives the Doppler spectrum after integration at range bin 79 , which is obtained from experimentally derived data with red dashed line denoting spectrum before filtering and blue solid line denoting spectrum after process. It can be observed that there are several peaks well above $-30 \mathrm{~dB}$ before filtering, which create an unfavorable ship detection environment. However, the ship target at Doppler bin 183 can be distinguished easily since the ionospheric clutters are suppressed by about $10 \mathrm{~dB}$ in average, whereas the target signal is undistorted.

Besides ship detection, the other focus can be drawn on the sea state remote sensing. A successful sea state inversion algorithm depends on the correct and accurate extractions of Bragg. In Figure 7, the double first order Bragg peaks at Doppler bins (in between the vertically green dashed lines) are contaminated by the ionospheric clutters. After GOP filtering, the contaminations of ionosphere are minimized; moreover the SINR of the negative Bragg peaks was raised around $12 \mathrm{~dB}$, which facilitates its extraction algorithms.

The Doppler spectrums from all the range bins are then collected together, forming a range-Doppler spectrum. Figure 8(a) presents the original range-Doppler spectrum which is contaminated by the ionospheric clutters from range bins 73 to 96 , whereas Figure 8 (b) describes the spectrum after being processed by GOP filters. Obviously, the ionospheric clutters which widely spread in range and Doppler bins are suppressed effectively. The average SINR improvement is above $10 \mathrm{~dB}$ in this experiment.

\section{Conclusions}

In this paper, a novel GOP with adjustable ISC parameter has been proposed. It is proved to be an extension of OP and four fundamental properties are presented with proofs. The construction of GOP filters is demonstrated with examples of spatial and polarization filtering, respectively. In the first example, with ISC derived from adaptive beamforming 


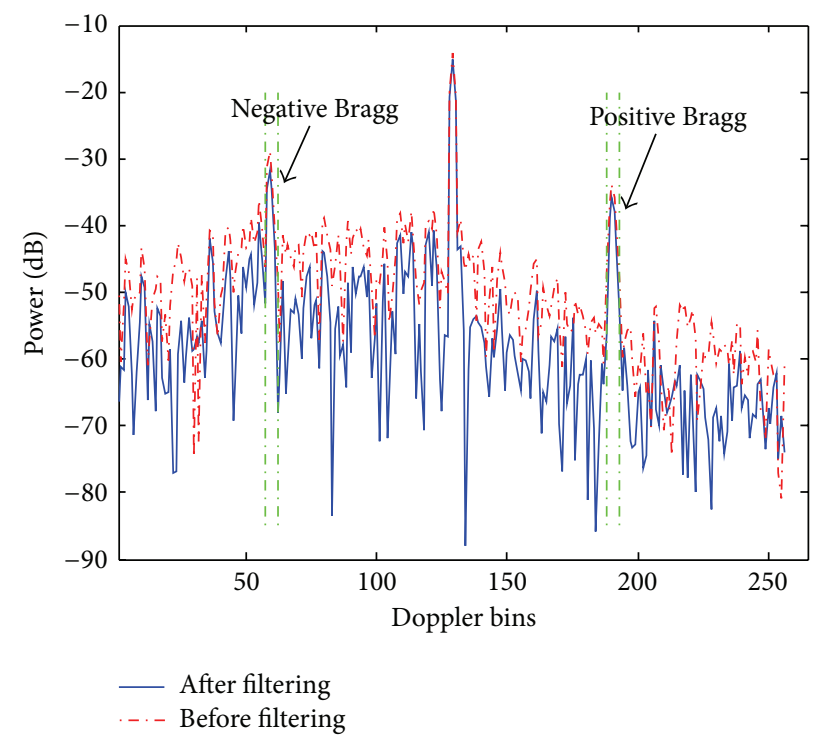

FIgURE 7: Doppler spectrum at range bin 89.

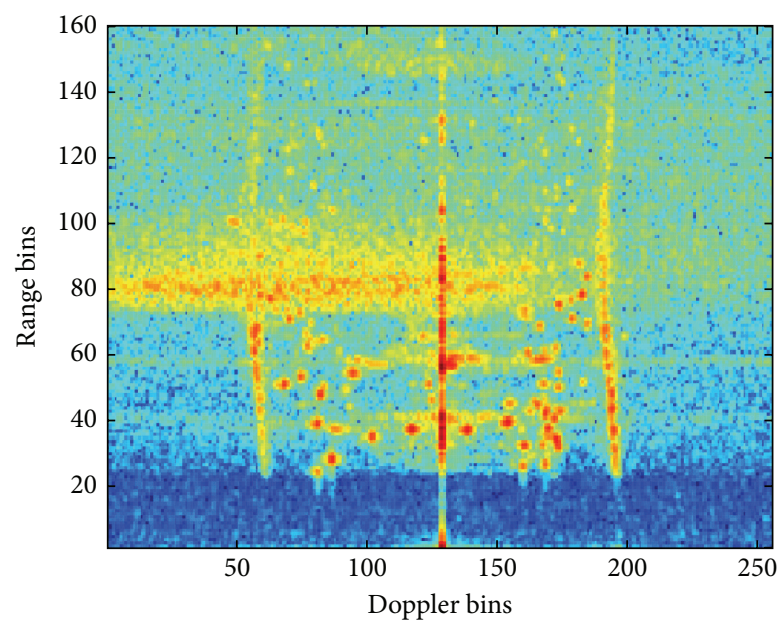

(a) Original range-Doppler spectrum

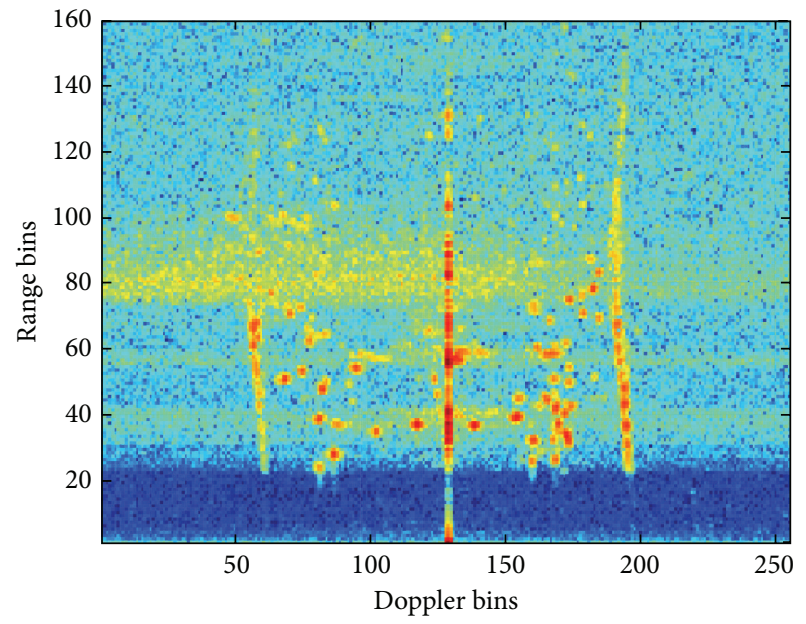

(b) Range-Doppler spectrum after GOP filter

FIGURE 8: Range-Doppler spectrum of HFSWR.

algorithm, the SINR performance of GOP filter is shown to be better than MVDR and OP filters both theoretically and numerically. A GOP polarization filter is constructed with manually designed ISC in the second example. An extra coherent loss is introduced to the ionospheric clutters by purpose and a satisfying clutter suppression result in HFSWR is achieved. Besides the examples given, more designs of GOP filter can be inspired by the flexibilities of ISC. It can be concluded that GOP filter is a promising filtering technique for interference suppression and capable of being used in spatial filtering, polarization filtering, and other array signal processing applications.

\section{Conflict of Interests}

The authors declare that there is no conflict of interests regarding the publication of this paper.

\section{Acknowledgments}

This project is sponsored by the National Marine Technology Program for Public Welfare (no. 200905029), National Natural Science Foundation of China (no. 61171180), and Team Research Foundation for Fundamental Research Program in FIO, China (no. GY0213T03).

\section{References}

[1] R. T. Behrens and L. L. Scharf, "Signal processing applications of oblique projection operators," IEEE Transactions on Signal Processing, vol. 42, no. 6, pp. 1413-1424, 1994.

[2] L. Rebollo-Neira, "Oblique matching pursuit," IEEE Signal Processing Letters, vol. 14, no. 10, pp. 703-706, 2007.

[3] G. W. Stewart, "On the numerical analysis of oblique projectors," SIAM Journal on Matrix Analysis and Applications, vol. 32, no. 1, pp. 309-348, 2011. 
[4] X.-P. Mao, A.-J. Liu, H.-J. Hou, H. Hong, R. Guo, and W.-B. Deng, "Oblique projection polarisation filtering for interference suppression in high-frequency surface wave radar," IET Radar, Sonar and Navigation, vol. 6, no. 2, pp. 71-80, 2012.

[5] X. P. Mao, A. J. Liu, W. B. Deng, B. Cao, and Q. Y. Zhang, "An oblique projecting polarization filter," Acta Electronica Sinica, vol. 38, no. 9, pp. 2003-2008, 2010 (Chinese).

[6] Q.-Y. Zhang, B. Cao, L. Jin, and N.-T. Zhang, "Oblique projection polarization filtering-based interference suppressions for radar sensor networks," EURASIP Journal on Wireless Communications and Networking, vol. 2010, Article ID 605103, 2010.

[7] X. Yu and L. Tong, "Joint channel and symbol estimation by oblique projections," IEEE Transactions on Signal Processing, vol. 49, no. 12, pp. 3074-3083, 2001.

[8] Q. Zhang, B. Cao, J. Wang, and N. Zhang, "Polarization filtering technique based on oblique projections," Science China F, vol. 53, no. 5, pp. 1056-1066, 2010.

[9] Y. Cui, K. Liu, and J. Wang, "Direction-of-arrival estimation for coherent GPS signals based on oblique projection," Signal Processing, vol. 92, no. 1, pp. 294-299, 2012.

[10] Y. Hu, X. Zhang, F. Zhu, and H. Lv, "Image recognition using iterative oblique projection," Electronics Letters, vol. 41, no. 20, pp. 1109-1110, 2005.

[11] G. Hellbourg, R. Weber, C. Capdessus, and A.-J. Boonstra, "Oblique projection beamforming for RFI mitigation in radio astronomy," in Proceedings of the IEEE Statistical Signal Processing Workshop (SSP '12), pp. 93-96, IEEE, August 2012.

[12] P. De, "New methods for sensing bandlimited signals in cognitive radio," in Proceedings of the IEEE 65th Vehicular Technology Conference (VTC '07-Spring), pp. 1916-1920, Dublin, Ireland, April 2007.

[13] R. Boyer, "Oblique projection for source estimation in a competitive environment: algorithm and statistical analysis," Signal Processing, vol. 89, no. 12, pp. 2547-2554, 2009.

[14] M. L. McCloud and L. L. Scharf, "A new subspace identification algorithm for high-resolution DOA estimation," IEEE Transactions on Antennas and Propagation, vol. 50, no. 10, pp. 13821390, 2002.

[15] R. Boyer and G. Bouleux, "Oblique projections for direction-ofarrival estimation with prior knowledge," IEEE Transactions on Signal Processing, vol. 56, no. 4, pp. 1374-1387, 2008.

[16] H. Hong, X.-P. Mao, C. Hu, R. Guo, W.-B. Deng, and P. Jiang, "Narrow-band null phase-shift spatial filter based on oblique projection," in Proceedings of the IEEE Radar Conference (RADAR '13), pp. 1-5, Ottawa, Canada, May 2013.

[17] Z. H. Xu, Y. P. Ni, and L. Jin, "Resolution theory of polarization sensitive array signals," in Proceedings of the CIE International Conference on Radar (ICR '06), pp. 1-4, IEEE, Shanghai, China, October 2006.

[18] X. P. Mao and Y. T. Liu, "Null phase-shift polarization filtering for high-frequency radar," IEEE Transactions on Aerospace and Electronic Systems, vol. 43, no. 4, pp. 1397-1408, 2007.

[19] Y.-M. Wang, X.-P. Mao, J. Zhang, and H. Hong, "A multidomain collaborative filter based on polarization sensitive frequency diverse array," in Proceedings of the IEEE Radar Conference, pp. 0507-0511, Cincinnati, Ohio, USA, May 2014.

[20] M. Souden, J. Benesty, and S. Affes, "A study of the LCMV and MVDR noise reduction filters," IEEE Transactions on Signal Processing, vol. 58, no. 9, pp. 4925-4935, 2010.
[21] Y. M. Cui, X. P. Mao, and H. Hong, "A novel adaptive filter for interference suppression," in Proceedings of the IEEE Region 10 Conference, vol. 31194, pp. 1-5, IEEE, 2013.

[22] H. C. Chan, "Characterization of ionospheric clutter in HF surface wave radar," Tech. Rep. 2003-114, Defence R\&D Canada, Ottawa, Canada, 2003. 

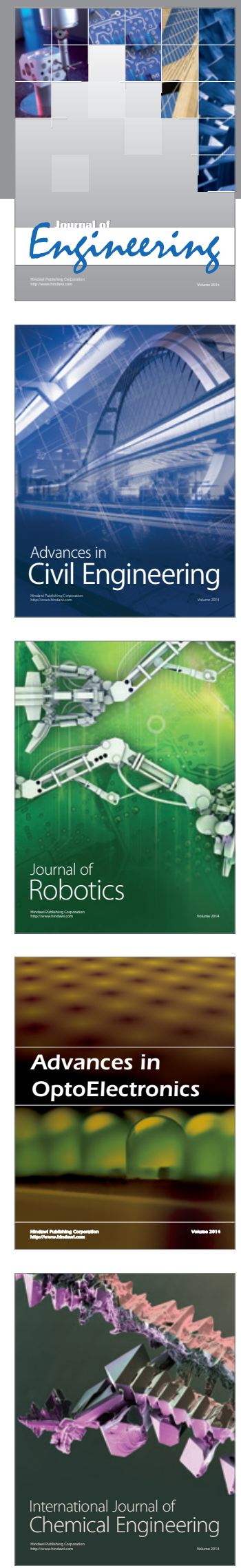

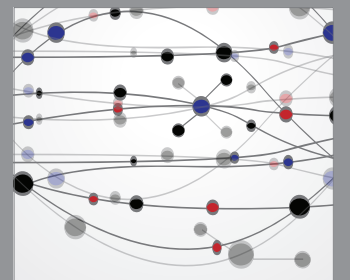

The Scientific World Journal
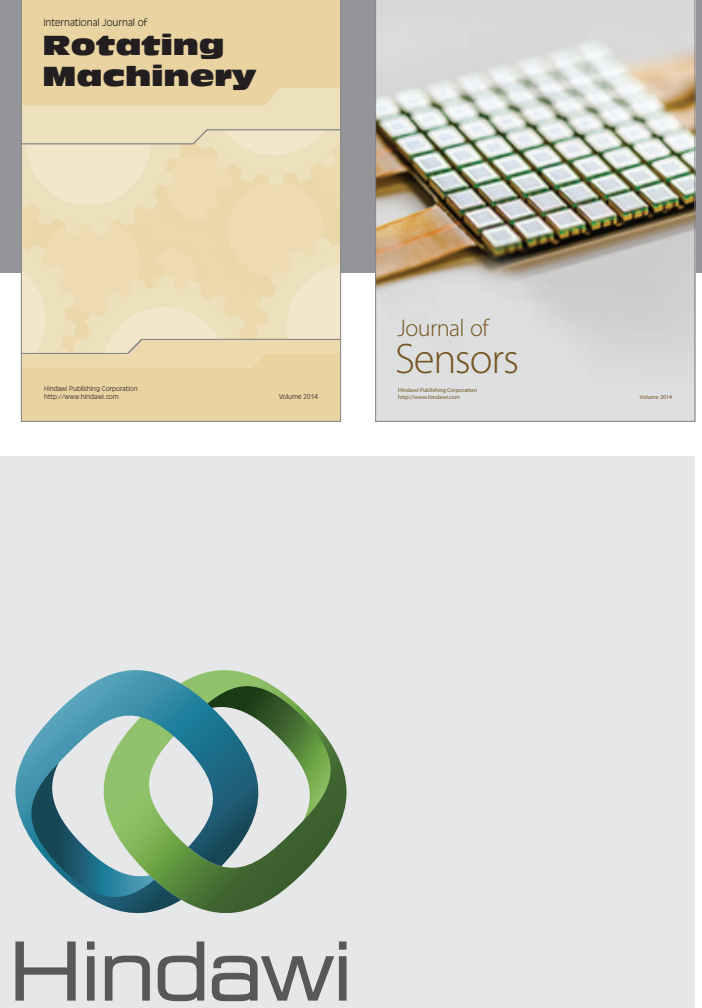

Submit your manuscripts at http://www.hindawi.com
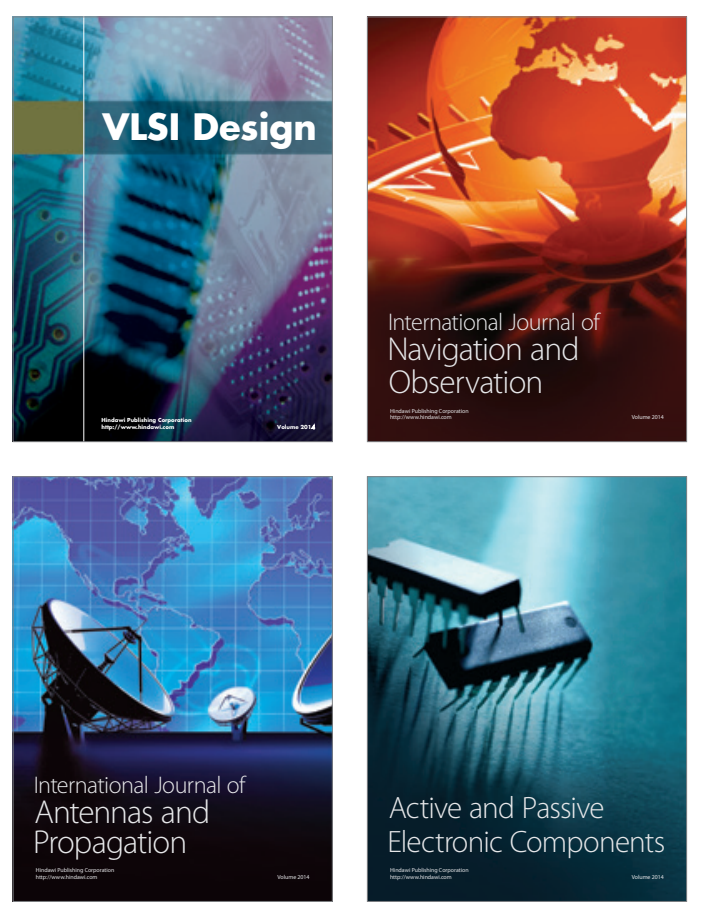
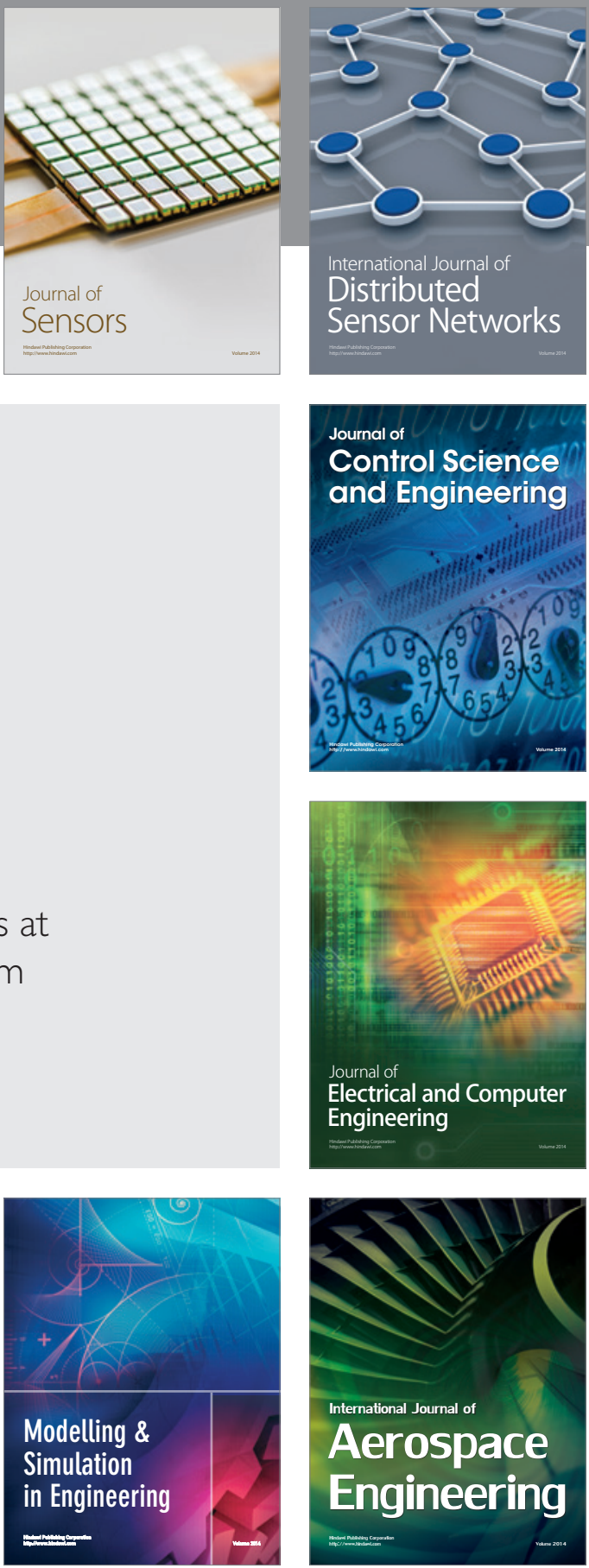

Journal of

Control Science

and Engineering
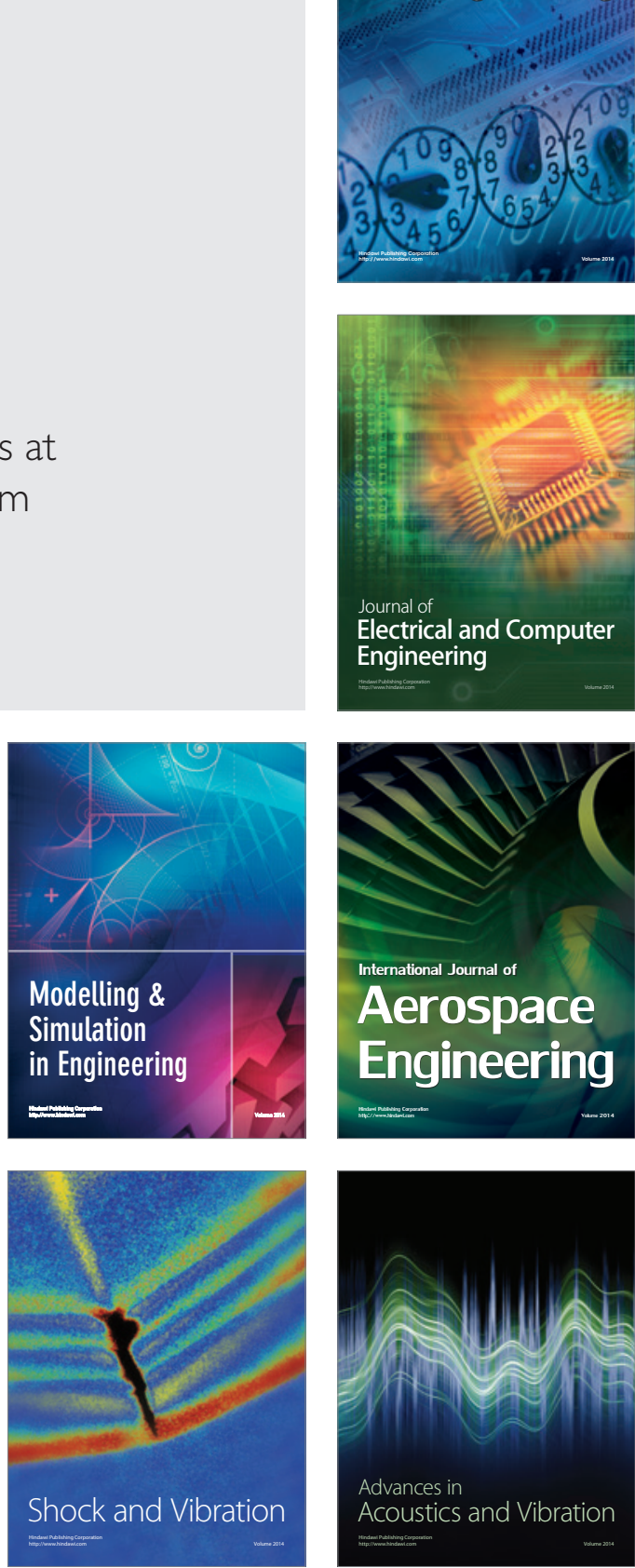